\title{
Beam Probe Imaging of Electron Density in the Chaotic Region of CHS
}

\author{
Harukazu IGUCHI, Kiichiro NAKAMURA, Josef SCHWEINZER ${ }^{1)}$ and CHS Group \\ National Institute for Fusion Science, Toki 509-5292 Japan \\ 1) Max-Planck-Institut fur Plasmaphysik, D-4086 Garching bei Munchen, Germany
}

(Received 4 December 2006 / Accepted 26 April 2007)

\begin{abstract}
A neutral lithium beam probe (LiBP) for the Compact Helical System (CHS) has been extended to a twodimensional imaging diagnostic (2-D-LiBP). Two-dimensional image of the plasma structure in the edge and separatrix region of the $l=2$ helical device is obtained by changing the beam injection angle mechanically, shotto-shot. The 2-D image has shown the asymmetric plasma structure in the edge separatrix region, suggesting that 2-D diagnostic is essential in the chaotic magnetic field region where magnetic surfaces are not defined by a flux function.

(c) 2007 The Japan Society of Plasma Science and Nuclear Fusion Research
\end{abstract}

Keywords: lithium beam, imaging diagnostic, beam probe imaging, edge plasma modeling

DOI: $10.1585 /$ pfr.2.S1102

\section{Inroduction}

Imaging diagnostic methods are widely used in many fields of science. We can derive information or insight more from a visualized image than from simple onedimensional graphic data. The most popular example is the computed tomography (CT) image using X-ray for medical diagnostic. In the field of plasma diagnostics, the $\mathrm{CT}$ methods have been adopted for soft X-ray or electron cyclotron emission (ECE) diagnostics to analyze MHD activities in tokamaks [1]. Generally in these diagnostics, a set of multi-channel detector arrays viewing from different direction is used. Two-dimensional images of plasma structure are reconstructed by the use of a numerical algorithm assuming certain kinds of symmetry. Resolution of the image depends on the number of detector arrays. CCD cameras sensitive to the wavelength range from infrared to soft X-ray are also popular, which can directly take the 2-D image of the objectives [2]. A bolometer camera is another example [3]. However, the camera image is the result of integration along the sight line. Spatial resolution, in general, is not adequate to interpret the image as a local plasma structure. In order to overcome this disadvantage, local gas puffing or thermal atom flux injection method is applied, where local emission of the injected atoms are detected [4]. However, observation area is limited to far edge region because of strong attenuation of the injected atoms due to slow atomic velocities.

Plasma diagnostics using accelerated particle beams have advantages of good beam penetration and high spatial resolution, because the observation point is localized at the crossing of the beam path and the sight line. A beam emission spectroscopy (BES) using a heating neutral beam [5] and a lithium neutral beam probe [6] are examples of such diagnostics. In general, they are mostly onedimensional diagnostics, because observation points lies along the fixed injection beam path. As for the BES, a twodimensional map of density fluctuations was successfully obtained at the DIII-D tokamak experiment, where observation points are aligned across the beam as well as along the beam $[5,7]$. The method can be referred to the first imaging diagnostic using the particle beam. Another example is the LiBP on ASDEX-U, where electro-static beam scanning is adopted to get a 2-D image of edge plasma density distribution in a single shot [8]. However, in both experiments, the observation areas are limited to small part of plasma cross sections.

In this paper, we introduce an extension of a normal one-dimensional LiBP to the two-dimensional imaging diagnostic, where a beam line with variable injection angle is used [9]. The observation area covers significant part of the edge chaotic magnetic field region of the helical device CHS. Since the beam injection angle is mechanically changed shot-to-shot, the 2-D image of the beam emission is obtained with multiple discharges of identical operational conditions. Although the method is not a single shot imaging at the moment, it has brought new information that cannot be obtained with one-dimensional measurement. It is noted that studies of plasma behaviors in an ergodic region is also important to improve the ergodic divertor concept proposed in TEXTOR [10] and/or DIIID [11] tokamaks. 2-D diagnostics will also contribute to such researches. 


\section{Description of 2-D-LiBP}

The LiBP has been used in many fusion devices especially for edge plasma diagnostics, because of its strong beam emission in the visible range. The cross-section for the LiI resonance line $(670.8 \mathrm{~nm})$ emission by electron impact is larger than that for the $\mathrm{H}_{\alpha}$ emission by three orders of magnitude. The beam with the current of $\mathrm{mA}$ level will be useful. In contrast, for the BES, heating neural beam with the current of tens amperes is used. In most of the LiBPs, thermionic ion sources have been used, because of its easiness in handling and small energy spread. It gives fine probe beams with high spatial resolution.

Lithium atoms injected into plasmas are excited by collisions with plasma particles, mostly with electrons in the edge region, and emit photons. The number of photons $N_{v}(x)$ detected by the optical system of solid angle $\Omega$, sampling volume $V$ and efficiency $\eta$ is expressed as

$$
N_{v p}(x)=n_{e}(x) n_{b}(x)\left\langle\sigma_{e m} v_{r}\right\rangle_{e f f}(\Omega / 4 \pi) V \eta,
$$

where $n_{e}(x)$ is the electron density, $n_{b}(x)$ is the lithium atom density, $\left\langle\sigma_{e m} v_{r}\right\rangle_{e f f}$ is the effective emission rate coefficient of the resonance line. The parameter $x$ is the distance along the beam. The $v_{r}$ is the relative velocity between the injected lithium atom and the plasma electrons that induce lithium atom excitation. It is noted that the local beam emission intensity is proportional to the local electron density at the observation point. However, the interpretation is not so simple, because other terms in the right hand of equation (1) also depend on the electron density. First, the beam density changes due to ionization loss in the plasma, which is expressed as

$$
n_{b}(x)=n_{b 0} \exp \left(-\int_{0}^{x} \frac{\left\langle\sigma_{i o n} v_{r}\right\rangle}{v_{b}} d x\right) .
$$

The integration is carried out from the point $x=0$, which is a fixed point outside the plasma, to the observation point $x$. The effective emission rate coefficient is a function of electron density and temperature. Generally for edge plasmas, ion impact excitation can be neglected. The emission intensity at $670.8 \mathrm{~nm}$ is proportional to the number of excited lithium atoms at $2 \mathrm{P}$ state. It is primarily determined by the balance between excitation from the ground state and spontaneous emission (with the life-time of $27 \mathrm{nsec}$ ). However, it is also affected by various loss processes from the $2 \mathrm{P}$ state such as ionization, charge exchange, excitation to upper levels, stimulated de-excitation to $2 \mathrm{~S}$ state etc. depending on the plasma density. The effective rate coefficient described above thus has to be calculated using collisional-radiative (CR) model.

The density reconstruction algorithm including the atomic level up to $n=4$ was developed by J. Schweinzer et al. [12]. More simple algorithm using effective rate coefficients was developed by S. Sasaki, et al., [13], and by K. Nakamura, et al., [7], in which electron temperature dependence is neglected. Since the electron impact excitation cross-section for the lithium atom is a weak function of the electron temperature in the range from ten to a few hundreds $\mathrm{eV}$, the assumption is reasonable for edge plasmas. For details on the density reconstruction, see those references. We use the latter method mostly in the experiments, because the information on electron temperature profile is not necessarily given for all region of the edge. Crosscheck and estimate of error caused by this simplification is performed occasionally with the help of Schweinzer's code. The electron density is underestimated where the electron temperature is low, for example, below $5 \mathrm{eV}$. But the region is limited far edge and it does not affect the experimental results here.

\section{Experiments}

The CHS is a low-aspect-ratio torus $\left(A_{p}\right.$ of 5$)$ with $l=2$ and $m=8$ helical windings. The major and minor radii are $1.0 \mathrm{~m}$ and $0.2 \mathrm{~m}$, respectively. The maximum magnetic field strength is $1.8 \mathrm{~T}$ on the axis. A hydrogen plasma is produced and heated by electron cyclotron resonance heating $(53 \mathrm{GHz} \mathrm{ECH} / 400 \mathrm{~kW})$ and neutral beam heating (40 kV NBIs/1.3 MW).

Figure 1 show the schematic arrangement of the 2-D$\mathrm{LiBP}$ on CHS. The beam injector with tilting mechanism is mounted on an upper port. The tilting angle is \pm 18 degrees. The lithium neutral beam at the energy of $15 \mathrm{keV}$ and the current of $0.1 \mathrm{~mA}$ is injected into the plasma. The beam diameter is about $20 \mathrm{~mm}$ in the CHS vacuum chamber. Light collection optics is located on the side port of the torus. The beam emission collected by the lens with a diameter of $10 \mathrm{~cm}$ (effective F-number about 4 ) is transmitted to 8-channel optical interference filters via plastic fibers and is introduced to Avalanche-Photo-Diode detectors (APD). The APDs are followed by pre-amplifiers with frequency response up to $100 \mathrm{kHz}$. Twenty-five couplers for optical fiber connection are prepared behind the light collection

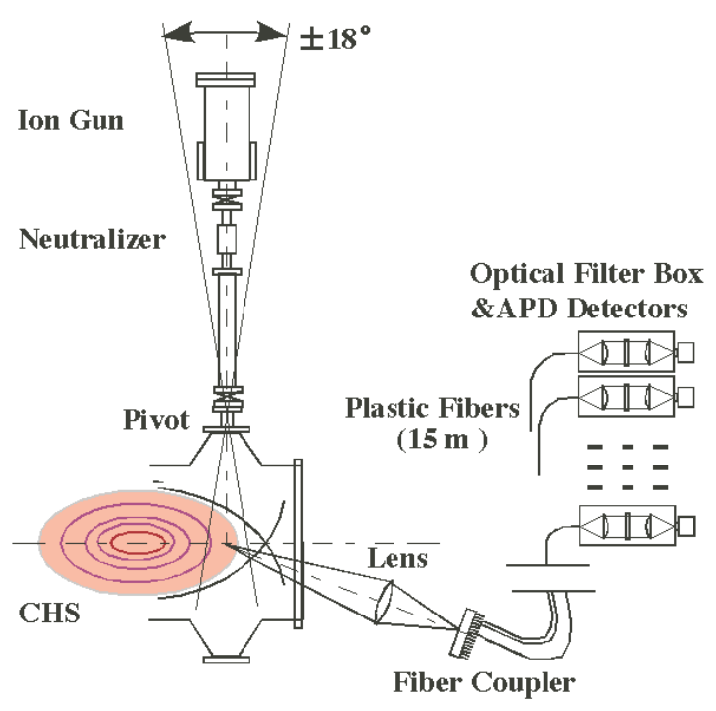

Fig. 1 Schematic of the 2-D-LiBP on CHS. 


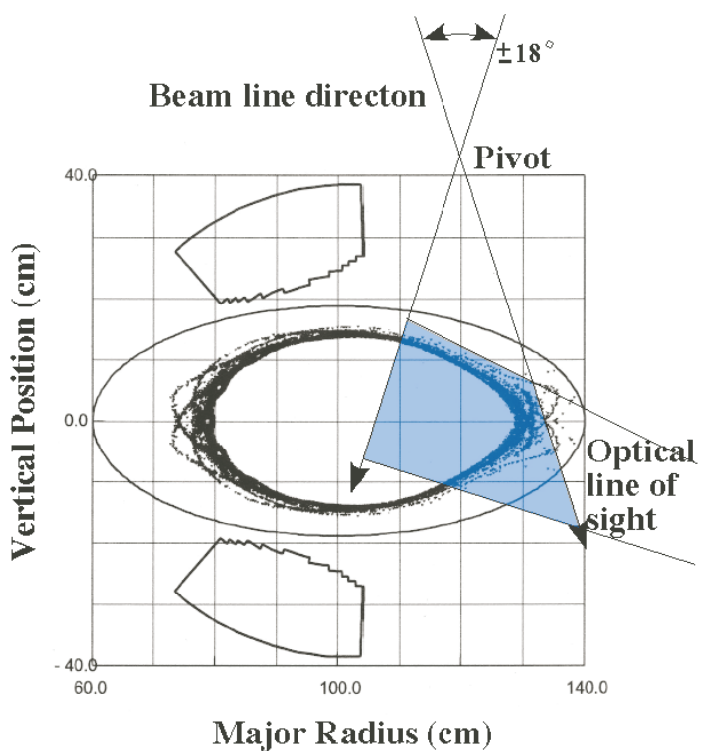

Fig. 2 Observation area superposed with chaotic magnetic field structure for the magnetic divertor configuration.

lens to cover the necessary observation area. The sampling length is $2 \mathrm{~mm}$ along the beam and the spacing between channels is $8 \mathrm{~mm}$. Thus the observation area covers significant part of the edge and separatrix region as shown in Fig. 2., where chaotic magnetic field structure outside the last closed flux surfaces (LCFS) is superposed.

Two-dimensional beam emission profiles are obtained by changing the beam injection angle shot-to shot. The beam is modulated at $4 \mathrm{kHz}$ for phase sensitive detection to improve signal to noise ratio. Its time constant is $3 \mathrm{~ms}$ in the present measurements, which gives time resolution about 10 msec.

\section{Experimental Results}

Experiments for the magnetic divertor configuration at the magnetic axis of $R_{\mathrm{ax}}=1.016 \mathrm{~m}$ and the magnetic field strength of $0.93 \mathrm{~T}$ is here introduced as an example of beam probe imaging. The average electron density for the target plasma is about $2 \times 10^{19} \mathrm{~m}^{-3}$ in the NBI plasma. Two-dimensional contour map of the beam emission intensity is shown in Fig. 3. The dots in the image indicate the observation points for the data set. The emission intensity increases as the beam penetrates into the plasma. It reaches maximum and then decreases as the beam further penetrate, which is due to the beam attenuation in the core plasma. Although the beam intensity changes gradually in time, it does not affect the density reconstruction calculation, because the beam intensity is calibrated shot-to-shot by detecting the beam emission at the gas puff phase just before the magnetic field is terminated [9]. The vertical position $y=0$ in the figure is the equatorial plane of the torus and $x=1275 \mathrm{~mm}$ corresponds to the position where the LCFS crosses the equatorial plane.

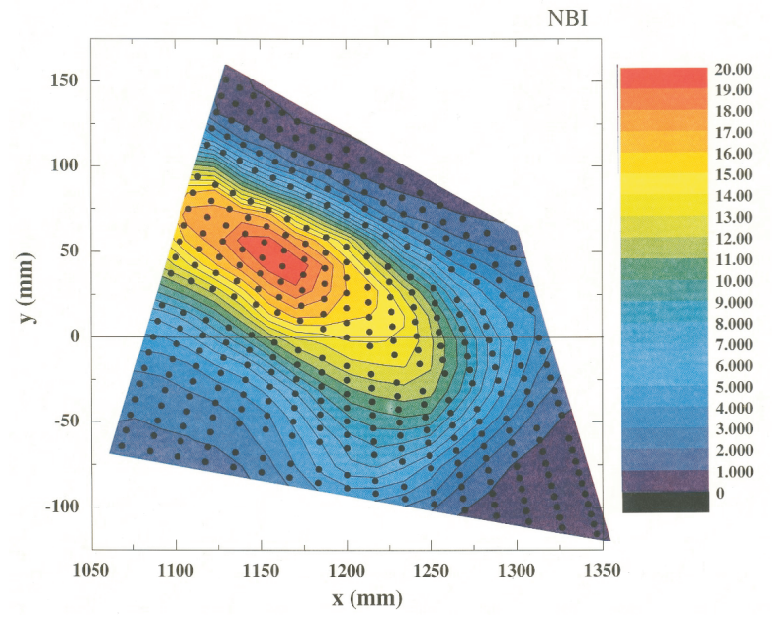

Fig. 3 Two-dimensional beam emission image for the NBI heated plasma.

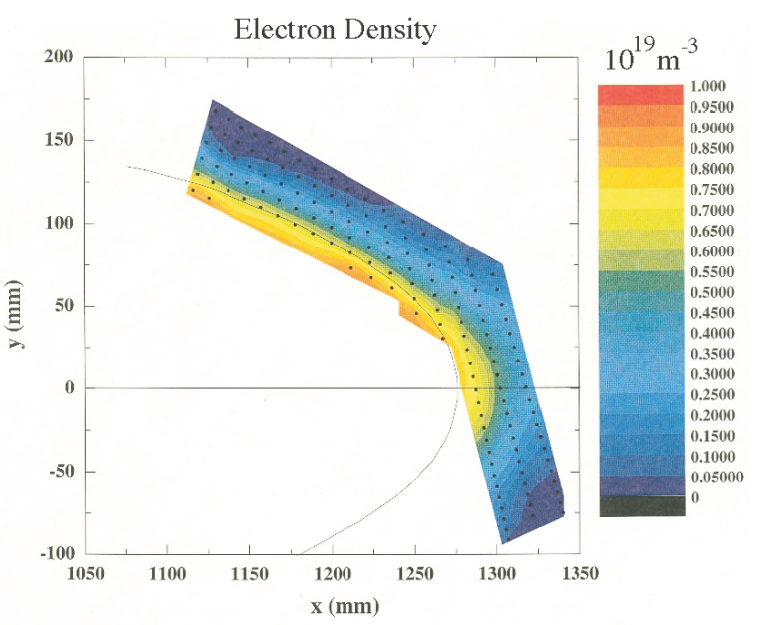

Fig. 4 Two-dimensional electron density profile reconstructed from the emission profile in Fig. 2.

The two-dimensional map of the edge density distribution is reconstructed from the emission data as shown in Fig. 4. Since the density reconstruction calculation loses accuracy in the core plasma region where beam attenuation is too strong, the reliable density profile is limited near and outside the LCFS, which is shown in the figure. It is suggested in the figure that the plasma shifts upward in the chaotic field region near the separatrix, although the chaotic magnetic field structure has up-down symmetry. The density peak shifts further upward as horizontal location goes outward. In order to see the effect of magnetic field direction on the plasma shifts, experiments for the reversed magnetic field direction have been carried out. It is shown that he electron density distribution now shifts downward. Comparison of vertical density profile in the two cases is shown in Fig. 5. The vertical scale is normalized for profile comparison. It is noted that the updown asymmetry is sustained in steady state. The observed 


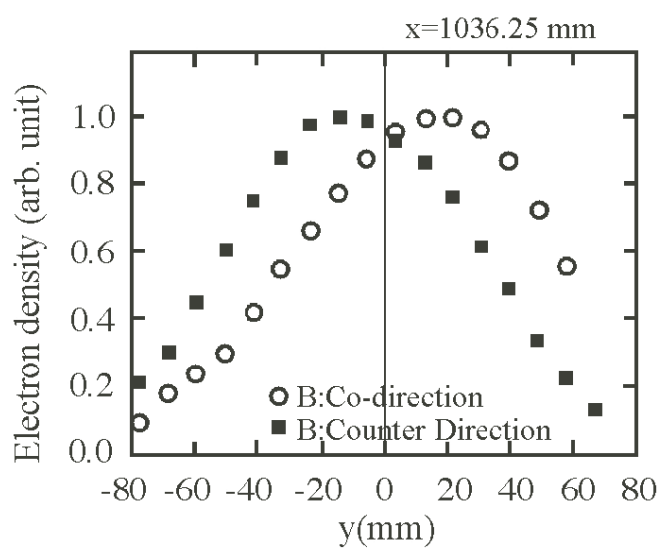

Fig. 5 The normalized electron density profiles along vertical line at $x=1325 \mathrm{~mm}$ for normal and reversed toroidal magnetic field.

asymmetry might be caused by the cross-field plasma flow. The experimental result suggests that the flow is in the direction of ion $\boldsymbol{B} \times \nabla B$ drift. Since the $\boldsymbol{B} \times \nabla B$ drift is directed opposite for ions and electrons, electric field will be induced. Then the $\boldsymbol{E} \times \boldsymbol{B}$ drift will affect the final flow patterns. The observed asymmetry will induce loss flux asymmetry on divertor plates, which might cause severe problem in heat removal from the divertor plates. In order to understand plasma behavior in the chaotic region, modeling of plasma in quasi-equilibrium including flow seems to be necessary, which is left for future study. Such study will contribute to ergodic divertor studies in tokamaks.

The spatial resolution of the measurements along the beam is about $10 \mathrm{~mm}$ or less. Since the $15 \mathrm{keV} \mathrm{Li}$ beam travels $17 \mathrm{~mm}$ during the life-time of $2 \mathrm{P}$ state, the beam emission shifts down stream of the beam from the electron location that contribute to beam excitation. In the present experiments, however, the electron density is around $10^{19} \mathrm{~m}^{-3}$ near the LCFS, where shortening of the life-time occurs due to the loss processes from the $2 \mathrm{P}$ state such as ionization, charge exchange, excitation to upper levels, stimulated de-excitation to $2 \mathrm{~S}$ state etc. Taking these rate coefficients into account [14], the spatial shift and blurring becomes smaller. It is less than $10 \mathrm{~mm}$ near the LCFS. It is noted that the spatial density distribution should deform toward up-stream in the low-density far edge region. Since the beam profile is parabolic and the FWHM is about $20 \mathrm{~mm}$, the effective spatial resolution across the beam is better than $20 \mathrm{~mm}$.

The temporal resolution of the measurement for each observation point is $10 \mathrm{msec}$ at the moment with beam current of $0.1 \mathrm{~mA}$. The time resolution will be improved by increasing the beam current. If the beam current is higher by two orders of magnitude, density fluctuations can even be measured as is done in the BES [5]. A lithium ion gun with the beam current of $10 \mathrm{~mA}$ is under development.

In summary, an imaging diagnostic combined with the lithium beam probing has been developed for the study of two-dimensional plasma structure in the chaotic magnetic field region of the helical device CHS. The 2-D map has shown an asymmetric plasma structure, which suggests the necessity of new modeling for plasma equilibrium in ergodic magnetic field region.

The research has been supported by the Grant-in-Aid of Science Research from the Japan Society for the Promotion of Science (JSPS), No. 16360461.

[1] Y. Nagayama et al., Phys. Rev. Lett. 69, 2376 (1992).

[2] S. Ohdachi et al., Plasma Sci. and Tech. 8, 45 (2006).

[3] B. Peterson et al., Plasma Physics and Controlled Fusion 45, 1167 (2003).

[4] S.J. Zweben et al., Phys. Plasmas 9, 1981 (2002).

[5] C. Fenzi et al., Rev. Sci. Instrum. 72, 988 (2001).

[6] D. Thomas, IEEE Trans. Plasma Science 24, 27 (1996).

[7] G.R. McKee et al., Rev. Sci. Instrum. 74, 2014 (2003).

[8] S. Zoletnik et al., Rev. Sci. Instrum. 76, 073504 (2005).

[9] K. Nakamura et al., Rev. Sci. Instrum. 76, 013504 (2005).

[10] K.H. Finken et al., Phys. Rev. Lett. 94, 015003 (2005).

[11] T.E. Evans et al., nature physics: ADVNCE ONLINE PUBLICATION, www.nature.com/naturephysics, 2006.

[12] J. Schweinzer et al., Plasma Phys. Control. Fusion 34, 1173 (1992).

[13] S. Sasaki et al., Rev. Sci. Instrum. 64, 1699 (1993).

[14] J. Schweinzer et al., Atomic Data Nucl. Data Tables 72, 239 (1999). 